\title{
Some New Lacunary Strong Convergent Vector-Valued Sequence Spaces
}

\author{
M. Mursaleen, ${ }^{1}$ A. Alotaibi, ${ }^{2}$ and Sunil K. Sharma ${ }^{3}$ \\ ${ }^{1}$ Department of Mathematics, Aligarh Muslim University, Aligarh 202002, India \\ ${ }^{2}$ Department of Mathematics, Faculty of Science, King Abdulaziz University, P.O. Box 80203, Jeddah 21589, Saudi Arabia \\ ${ }^{3}$ Department of Mathematics, Model Institute of Engineering \& Technology, Kot Bhalwal 181122, India
}

Correspondence should be addressed to M. Mursaleen; mursaleenm@gmail.com

Received 29 March 2014; Accepted 28 June 2014; Published 24 July 2014

Academic Editor: Hassan Eltayeb

Copyright (C) 2014 M. Mursaleen et al. This is an open access article distributed under the Creative Commons Attribution License, which permits unrestricted use, distribution, and reproduction in any medium, provided the original work is properly cited.

We introduce some vector-valued sequence spaces defined by a Musielak-Orlicz function and the concepts of lacunary convergence and strong $(A)$-convergence, where $A=\left(a_{i k}\right)$ is an infinite matrix of complex numbers. We also make an effort to study some topological properties and some inclusion relations between these spaces.

\section{Introduction and Preliminaries}

An Orlicz function $M:[0, \infty) \rightarrow[0, \infty)$ is convex and continuous such that $M(0)=0, M(x)>0$ for $x>0$. Lindenstrauss and Tzafriri [1] used the idea of Orlicz function to define the following sequence space:

$$
\ell_{M}=\left\{x \in w: \sum_{k=1}^{\infty} M\left(\frac{\left|x_{k}\right|}{\rho}\right)<\infty\right\},
$$

which is called as an Orlicz sequence space. The space $\ell_{M}$ is a Banach space with the norm

$$
\|x\|=\inf \left\{\rho>0: \sum_{k=1}^{\infty} M\left(\frac{\left|x_{k}\right|}{\rho}\right) \leq 1\right\} .
$$

It is shown in [1] that every Orlicz sequence space $\ell_{M}$ contains a subspace isomorphic to $\ell_{p}(p \geq 1)$. An Orlicz function $M$ satisfies $\Delta_{2}$-condition if and only if, for any constant $L>1$, there exists a constant $K(L)$ such that $M(L u) \leq K(L) M(u)$ for all values of $u \geq 0$. An Orlicz function $M$ can always be represented in the following integral form:

$$
M(x)=\int_{0}^{x} \eta(t) d t
$$

where $\eta$ is known as the kernel of $M$ and is right differentiable for $t \geq 0, \eta(0)=0, \eta(t)>0 ; \eta$ is nondecreasing and $\eta(t) \rightarrow$ $\infty$ as $t \rightarrow \infty$.
A sequence $\mathscr{M}=\left(M_{k}\right)$ of Orlicz functions is called a Musielak-Orlicz function; see $([2,3])$. A sequence $\mathcal{N}=\left(N_{k}\right)$ defined by

$$
N_{k}(v)=\sup \left\{|v| u-M_{k}(u): u \geq 0\right\}, \quad k=1,2, \ldots
$$

is called the complementary function of a Musielak-Orlicz function $\mathscr{M}$. For a given Musielak-Orlicz function $\mathscr{M}$, the Musielak-Orlicz sequence space $t_{\mathscr{M}}$ and its subspace $h_{\mathscr{M}}$ are defined as follows:

$$
\begin{gathered}
t_{\mathscr{M}}=\left\{x \in w: I_{\mathscr{M}}(c x)<\infty \text { for some } c>0\right\}, \\
h_{\mathscr{M}}=\left\{x \in w: I_{\mathscr{M}}(c x)<\infty, \forall c>0\right\},
\end{gathered}
$$

where $I_{\mathscr{M}}$ is a convex modular defined by

$$
I_{\mathscr{M}}(x)=\sum_{k=1}^{\infty} M_{k}\left(x_{k}\right), \quad x=\left(x_{k}\right) \in t_{\mathscr{M}} .
$$

We consider $t_{\mathscr{M}}$ equipped with the Luxemburg norm

$$
\|x\|=\inf \left\{k>0: I_{\mathscr{M}}\left(\frac{x}{k}\right) \leq 1\right\}
$$

or equipped with the Orlicz norm

$$
\|x\|^{0}=\inf \left\{\frac{1}{k}\left(1+I_{\mathscr{M}}(k x)\right): k>0\right\} .
$$


A Musielak-Orlicz function $\left(M_{k}\right)$ is said to satisfy $\Delta_{2}$ condition if there exist constants $a, K>0$ and a sequence $c=\left(c_{k}\right)_{k=1}^{\infty} \in \ell_{+}^{1}$ (the positive cone of $\ell^{1}$ ) such that the inequality

$$
M_{k}(2 u) \leq K M_{k}(u)+c_{k}
$$

holds for all $k \in \mathbb{N}$ and $u \in R_{+}$, whenever $M_{k}(u) \leq a$.

Let $X$ be a linear metric space. A function $p: X \rightarrow \mathbb{R}$ is called paranorm, if

(1) $p(x) \geq 0$, for all $x \in X$;

(2) $p(-x)=p(x)$, for all $x \in X$;

(3) $p(x+y) \leq p(x)+p(y)$, for all $x, y \in X$;

(4) if $\left(\sigma_{n}\right)$ is a sequence of scalars with $\sigma_{n} \rightarrow \sigma$ as $n \rightarrow$ $\infty$ and $\left(x_{n}\right)$ is a sequence of vectors with $p\left(x_{n}-x\right) \rightarrow$ 0 as $n \rightarrow \infty$, then $p\left(\sigma_{n} x_{n}-\sigma x\right) \rightarrow 0$ as $n \rightarrow$ $\infty$.

A paranorm $p$ for which $p(x)=0$ implies $x=0$ is called total paranorm and the pair $(X, p)$ is called a total paranormed space. It is well known that the metric of any linear metric space is given by some total paranorm (see [4], Theorem 10.4.2, P-183). For more details about sequence spaces, see [5-11] and references therein.

The space of lacunary strong convergence has been introduced by Freedman et al. [12]. A sequence of positive integers $\theta=\left(k_{r}\right)$ is called "lacunary" if $k_{0}=0,0<k_{r}<k_{r+1}$ and $h_{r}=k_{r}-k_{r-1} \rightarrow \infty$, as $r \rightarrow \infty$. The intervals determined by $\theta$ are denoted by $I_{r}=\left(k_{r-1}, k_{r}\right]$ and the ratio $k_{r} / k_{r-1}$ will be denoted by $q_{r}$. The space of lacunary strongly convergent sequences $N_{\theta}$ is defined by Freedman et al. [12] as follows:

$$
N_{\theta}=\left\{x=\left(x_{i}\right): \lim _{r \rightarrow \infty} \frac{1}{h_{r}} \sum_{i \in I_{r}}\left|x_{i}-s\right|=0 \text {, for some } s\right\} .
$$

The space $\left|\sigma_{1}\right|$ of strongly Cesàro summable sequences is

$$
\begin{array}{r}
\left|\sigma_{1}\right|=\left\{x=\left(x_{k}\right): \text { there exists } L\right. \text { such that } \\
\left.\qquad \frac{1}{n} \sum_{i=1}^{n}\left|x_{i}-L\right| \longrightarrow 0, \text { as } n \longrightarrow \infty\right\} .
\end{array}
$$

In case, when $\theta=\left(2^{r}\right), N_{\theta}=\left|\sigma_{1}\right|$. Recently, Bilgin [13] in his paper generalized the concept of lacunary convergence and introduced the space $N_{0}(A, f)$, as

$$
\begin{aligned}
& N_{0}(A, f) \\
& =\left\{x=\left(x_{i}\right): \lim _{r \rightarrow \infty} \frac{1}{h_{r}} \sum_{i \in I_{r}} f\left(\left|A_{i}(x)-s\right|\right)=0, \text { for some } s\right\},
\end{aligned}
$$

where $f$ is a modulus function and $A=\left(A_{i}(x)\right) ; A_{i} x=$ $\sum_{k=1}^{\infty} a_{i k} x_{k}$ converges for each $i$. Later Bilgin [14] generalized lacunary strongly $A$-convergent sequences with respect to a sequence of modulus function $F=\left(f_{i}\right)$ as follows:

$$
\begin{aligned}
& N_{0}(A, F) \\
& =\left\{x=\left(x_{i}\right): \lim _{r \rightarrow \infty} \frac{1}{h_{r}} \sum_{i \in I_{r}} f_{i}\left(\left|A_{i}(x)-s\right|\right)=0, \text { for some } s\right\} .
\end{aligned}
$$

We write $\theta$ for the zero sequences.

Mursaleen and Noman [15] introduced the notion of $\lambda$ convergent and $\lambda$-bounded sequences as follows.

Let $\lambda=\left(\lambda_{k}\right)_{k=1}^{\infty}$ be a strictly increasing sequence of positive real numbers tending to infinity, that is,

$$
0<\lambda_{0}<\lambda_{1}<\cdots, \quad \lambda_{k} \longrightarrow \infty \quad \text { as } k \longrightarrow \infty,
$$

and said that a sequence $x=\left(x_{k}\right) \in w$ is $\lambda$-convergent to the number $L$, called the $\lambda$-limit of $x$ if $\Lambda_{m}(x) \rightarrow L$ as $m \rightarrow \infty$, where

$$
\lambda_{m}(x)=\frac{1}{\lambda_{m}} \sum_{k=1}^{m}\left(\lambda_{k}-\lambda_{k-1}\right) x_{k}
$$

The sequence $x=\left(x_{k}\right) \in w$ is $\lambda$-bounded if $\sup _{m}\left|\Lambda_{m}(x)\right|<$ $\infty$. It is well known [15] that if $\lim _{m} x_{m}=a$ in the ordinary sense of convergence, then

$$
\lim _{m}\left(\frac{1}{\lambda_{m}}\left(\sum_{k=1}^{m}\left(\lambda_{k}-\lambda_{k-1}\right)\left|x_{k}-a\right|\right)\right)=0
$$

This implies that

$$
\lim _{m}\left|\Lambda_{m}(x)-a\right|=\lim _{m}\left|\frac{1}{\lambda_{m}} \sum_{k=1}^{m}\left(\lambda_{k}-\lambda_{k-1}\right)\left(x_{k}-a\right)\right|=0
$$

which yields that $\lim _{m} \Lambda_{m}(x)=a$ and hence $x=\left(x_{k}\right) \in w$ is $\lambda$-convergent to $a$.

We now introduce the concept of lacunary strongly $A$ convergence for sequences with the elements chosen from a Banach space $(E,\|\cdot\|)$ over the complex field $\mathbb{C}$, with respect to Musielak-Orlicz functions $\mathscr{M}=\left(M_{i}\right)$.

Let $A=\left(a_{i k}\right)$ be an infinite matrix of complex numbers and $\mathscr{M}=\left(M_{i}\right)$ be a Musielak-Orlicz function. In the present paper we define the following sequence spaces:

$$
\begin{aligned}
N_{\theta}(E, A, \Lambda, \mathscr{M}) \\
=\left\{x=\left(x_{k}\right): x_{k} \in E,\right. \\
\quad \lim _{r \rightarrow \infty} \frac{1}{h_{r}} \sum_{i \in I_{r}}\left[M_{i}\left(\frac{\left\|A_{i}\left(\Lambda_{i}(x)-s_{i} e_{i}\right)\right\|}{\rho^{(i)}}\right)\right]=0
\end{aligned}
$$




$$
\begin{aligned}
& N_{\theta}^{0}(E, A, \Lambda, \mathscr{M}) \\
& =\left\{x=\left(x_{k}\right): x_{k} \in E,\right. \\
& \quad \lim _{r \rightarrow \infty} \frac{1}{h_{r}} \sum_{i \in I_{r}}\left[M_{i}\left(\frac{\left\|A_{i}\left(\Lambda_{i}(x)\right)\right\|}{\rho^{(i)}}\right)\right]=0 \\
& \left.\quad \text { for some } \rho^{(i)}>0\right\} .
\end{aligned}
$$

If we take $M_{i}(x)=x$, for all $i \in \mathbb{N}$, we have

$$
\begin{aligned}
& N_{\theta}(E, A, \Lambda) \\
& =\left\{x=\left(x_{k}\right): x_{k} \in E,\right. \\
& \lim _{r \rightarrow \infty} \frac{1}{h_{r}} \sum_{i \in I_{r}}\left[\frac{\left\|A_{i}\left(\Lambda_{i}(x)-s_{i} e_{i}\right)\right\|}{\rho^{(i)}}\right]=0 \\
& \text { for some } s=\left(s_{1}, s_{2}, \ldots\right) \in E, e_{i} \in \mathbb{C}, \\
& N_{\theta}^{0}(E, A, \Lambda) \\
& =\left\{x=\left(x_{k}\right): x_{k} \in E,\right. \\
& \lim _{r \rightarrow \infty} \frac{1}{h_{r}} \sum_{i \in I_{r}}\left[\frac{\left\|A_{i}\left(\Lambda_{i}(x)\right)\right\|}{\rho^{(i)}}\right]=0 \\
& \text { for some } \left.\rho^{(i)}>0\right\},
\end{aligned}
$$$$
\text { for some } \left.s=\left(s_{1}, s_{2}, \ldots\right) \in E, e_{i} \in \mathbb{C}, \rho^{(i)}>0\right\} \text {, }
$$

where

$$
e_{i}= \begin{cases}1, & \text { at the } i \text { th place } \\ 0, & \text { otherwise }\end{cases}
$$

A sequence $x$ is said to be $\Lambda$-lacunary strong $A$-convergent with respect to $\mathscr{M}$ if there is a number $s=\left(s_{1}, s_{2}, \ldots\right) \in E$, such that $x \in N_{\theta}(E, A, \Lambda, \mathscr{M})$.

We have generalized the strongly Cesàro-summable sequence space into $\Lambda$-strongly Cesàro-summable vectorvalued sequence space as

$$
\begin{aligned}
& \left|\sigma_{1}(E, A, \Lambda)\right| \\
& =\left\{x=x_{k}:\right. \\
& \text { there exists } L=\left(L_{1}, L_{2}, \ldots\right) \in E, e_{i} \in \mathbb{C} \\
& \text { such that } \left.\frac{1}{n} \sum_{i=1}^{n}\left\|A_{i}\left(\Lambda_{i}(x)-L_{i} e_{i}\right)\right\| \longrightarrow 0\right\},
\end{aligned}
$$

where $A=\left(a_{n k}\right)$ is a Cesàro matrix, that is,

$$
a_{n k}= \begin{cases}\frac{1}{n}, & \text { if } 1 \leq k \leq n, \\ 0, & \text { if } k \geq n .\end{cases}
$$

Then it can be shown that $\left|\sigma_{1}(E, A, \Lambda)\right|$ is a paranormed space with respect to the paranorm

$$
\|x\|=\left\|x_{1}\right\|+\sup _{n}\left(\frac{1}{n} \sum_{i=1}^{n}\left\|A_{i}\left(\Lambda_{i}(x)\right)\right\|\right) .
$$

\section{Topological Properties of the Spaces $N_{\theta}(E, A, \Lambda, \mathscr{M})$ and $N_{\theta}^{0}(E, A, \Lambda, \mathscr{M})$}

Theorem 1. Let $A=\left(a_{i k}\right)$ be an infinite matrix of complex numbers and let $\mathscr{M}=\left(M_{i}\right)$ be a Musielak-Orlicz function. Then $N_{\theta}(E, A, \Lambda, \mathscr{M})$ and $N_{\theta}^{0}(E, A, \Lambda, \mathscr{M})$ are linear spaces over the field of complex number $\mathbb{C}$.

Proof. It is easy to prove.

Theorem 2. Let $A=\left(a_{i k}\right)$ be an infinite matrix of complex numbers and let $\mathscr{M}=\left(M_{i}\right)$ be a Musielak-Orlicz function. Then $N_{\theta}^{0}(E, A, \Lambda, \mathscr{M})$ is normal spaces, when $E$ is normal.

Proof. Let $x \in N_{\theta}^{0}(E, A, \Lambda, \mathscr{M})$. Let $\|y\| \leq\|x\|$. Then

$$
\left\|A_{i}\left(\Lambda_{i}(y)\right)\right\| \leq\left\|A_{i}\left(\Lambda_{i}(x)\right)\right\| .
$$

Since $\mathscr{M}=\left(M_{i}\right)$ is increasing,

$$
\begin{aligned}
\frac{1}{h_{r}} \sum_{i \in I_{r}} & {\left[M_{i}\left(\frac{\left\|A_{i}\left(\Lambda_{i}(y)\right)\right\|}{\rho^{(i)}}\right)\right] } \\
& \leq \frac{1}{h_{r}} \sum_{i \in I_{r}}\left[M_{i}\left(\frac{\left\|A_{i}\left(\Lambda_{i}(x)\right)\right\|}{\rho^{(i)}}\right)\right] .
\end{aligned}
$$

Consequently, $y \in N_{\theta}^{0}(E, A, \Lambda, \mathscr{M})$. This completes the proof of the theorem.

Theorem 3. The spaces $N_{\theta}(E, A, \Lambda, \mathscr{M})$ and $N_{\theta}^{0}(E, A, \Lambda, \mathscr{M})$ are paranormed spaces, with respect to the paranorm

$$
\begin{gathered}
\|x\|=\inf \left\{\rho^{(i)}>0: M_{i}\left(\frac{\left\|a_{i 0} x_{1}\right\|}{\rho^{(i)}}\right)\right. \\
+\sup _{r \geq 1} \frac{1}{h_{r}} \sum_{i \in I_{r}}\left[M_{i}\left(\frac{\left\|A_{i}\left(\Lambda_{i}(x)\right)\right\|}{\rho^{(i)}}\right)\right] \leq 1, \\
\left.\rho^{(i)} \geq 0\right\} .
\end{gathered}
$$

Proof. It is easy to prove, so we omit the details.

\section{Relation between the Spaces $N_{\theta}(E, A, \Lambda)$ and $N_{\theta}(E, A, \Lambda, \mathscr{M})$}

The main purpose of this section is to study relation between $N_{\theta}(E, A, \Lambda)$ and $N_{\theta}(E, A, \Lambda, \mathscr{M})$.

Theorem 4. Let $A=\left(a_{i k}\right)$ be an infinite matrix of complex numbers and let $\mathscr{M}=\left(M_{i}\right)$ be a Musielak-Orlicz function 
satisfying $\Delta_{2}$-condition. If $x$ is $\Lambda$-lacunary strong $A$-convergent to $s$, with respect to $M$ and $(E,\|\cdot\|)$ is a normal Banach space, then $N_{\theta}(E, A, \Lambda) \subset N_{\theta}(E, A, \Lambda, \mathscr{M})$.

Proof. Let $x \in N_{\theta}(E, A, \Lambda)$ and $x \stackrel{\Lambda}{\rightarrow} s$, where $s=\left(s_{1}, s_{2}\right.$, $\ldots) \in E, e_{i} \in \mathbb{C}$. Then

$$
\lim _{r \rightarrow \infty} \frac{1}{h_{r}} \sum_{i \in I_{r}}\left(\frac{\left\|A_{i}\left(\Lambda_{i}(x)-s_{i} e_{i}\right)\right\|}{\rho}\right)=0 \quad \text { for some } \rho>0 .
$$

We define two sequences $y$ and $z$ such that

$$
\begin{aligned}
& \left(\left\|A_{i}\left(\Lambda_{i}(y)-s_{i} e_{i}\right)\right\|\right) \\
& = \begin{cases}\left(\left\|A_{i}\left(\Lambda_{i}(x)-s_{i} e_{i}\right)\right\|\right), & \text { if }\left(\left\|A_{i}\left(\Lambda_{k}(x)-s_{i} e_{i}\right)\right\|\right)>1, \\
\theta, & \text { if }\left(\left\|A_{i}\left(\Lambda_{i}(x)-s_{i} e_{i}\right)\right\|\right) \leq 1,\end{cases} \\
& \left(\left\|A_{i}\left(\Lambda_{i}(z)-s_{i} e_{i}\right)\right\|\right) \\
& = \begin{cases}\theta, & \text { if }\left(\left\|A_{i}\left(\Lambda_{i}(x)-s_{i} e_{i}\right)\right\|\right)>1, \\
\left(\left\|A_{i}\left(\Lambda_{i}(x)-s_{i} e_{i}\right)\right\|\right), & \text { if }\left(\left\|A_{i}\left(\Lambda_{k}(x)-s_{i} e_{i}\right)\right\|\right) \leq 1 .\end{cases}
\end{aligned}
$$

Hence,

$$
\begin{aligned}
\left(\left\|A_{i}\left(\Lambda_{i}(x)-s_{i} e_{i}\right)\right\|\right)= & \left(\left\|A_{i}\left(\Lambda_{i}(y)-s_{i} e_{i}\right)\right\|\right) \\
& +\left(\left\|A_{i}\left(\Lambda_{i}(z)-s_{i} e_{i}\right)\right\|\right) .
\end{aligned}
$$

Now,

$$
\begin{gathered}
\left(\left\|A_{i}\left(\Lambda_{i}(y)-s_{i} e_{i}\right)\right\|\right) \leq\left(\left\|A_{i}\left(\Lambda_{i}(x)-s_{i} e_{i}\right)\right\|\right), \\
\left(\left\|A_{i}\left(\Lambda_{i}(z)-s_{i} e_{i}\right)\right\|\right) \leq\left(\left\|A_{i}\left(\Lambda_{i}(x)-s_{i} e_{i}\right)\right\|\right) .
\end{gathered}
$$

Since $N_{\theta}(E, A, \Lambda)$ is normal, $y, z \in N_{\theta}(E, A, \Lambda)$. Let $\sup _{i} M_{i}(2)=$ $T$. Then

$$
\begin{aligned}
\frac{1}{h_{r}} \sum_{i \in I_{r}}\left[M_{i}\left(\frac{\left\|A_{i}\left(\Lambda_{i}(x)-s_{i} e_{i}\right)\right\|}{\rho^{(i)}}\right)\right] \\
=\frac{1}{h_{r}} \sum_{i \in I_{r}}\left[M_{i}\left(\frac{\left\|A_{i}\left(\Lambda_{i}(y)-s_{i} e_{i}\right)\right\|+\left\|A_{i}\left(\Lambda_{i}(z)-s_{i} e_{i}\right)\right\|}{\rho^{(i)}}\right)\right] \\
\leq \frac{1}{h_{r}} \sum_{i \in I_{r}}\left[\frac{1}{2} M_{i}\left(2 \frac{\left\|A_{i}\left(\Lambda_{i}(y)-s_{i} e_{i}\right)\right\|}{\rho^{(i)}}\right)\right. \\
\left.+\frac{1}{2} M_{i}\left(2 \frac{\left\|A_{i}\left(\Lambda_{i}(z)-s_{i} e_{i}\right)\right\|}{\rho^{(i)}}\right)\right]
\end{aligned}
$$

$$
\begin{aligned}
& <\frac{1}{2} \frac{1}{h_{r}} \sum_{i \in I_{r}} K_{1}\left(\frac{\left\|A_{i}\left(\Lambda_{i}(y)-s_{i} e_{i}\right)\right\|}{\rho^{(i)}}\right) M_{i}(2) \\
& +\frac{1}{2} \frac{1}{h_{r}} \sum_{i \in I_{r}} K_{2}\left(\frac{\left\|A_{i}\left(\Lambda_{i}(z)-s_{i} e_{i}\right)\right\|}{\rho^{(i)}}\right) M_{i}(2) \\
& \leq \frac{1}{2} \frac{1}{h_{r}} \sum_{i \in I_{r}} K_{1}\left(\frac{\left\|A_{i}\left(\Lambda_{i}(y)-s_{i} e_{i}\right)\right\|}{\rho^{(i)}}\right) \sup M_{i}(2) \\
& +\frac{1}{2} \frac{1}{h_{r}} \sum_{i \in I_{r}} K_{2}\left(\frac{\left\|A_{i}\left(\Lambda_{i}(z)-s_{i} e_{i}\right)\right\|}{\rho^{(i)}}\right) \sup M_{i}(2) \\
& \longrightarrow 0 \quad \text { as } r \longrightarrow \infty .
\end{aligned}
$$

Hence $x \in N_{\theta}(E, A, \Lambda, \mathscr{M})$. This completes the proof of the theorem.

Theorem 5. Let $A=\left(a_{i k}\right)$ be an infinite matrix of complex numbers and let $\mathscr{M}=\left(M_{i}\right)$ be a Musielak-Orlicz function satisfying $\Delta_{2}$-condition. If

$$
\lim _{u \rightarrow \infty} \inf _{i} \frac{M_{i}\left(v / \rho^{(i)}\right)}{v / \rho^{(i)}}>0 \quad \text { for some } \rho^{(i)}>0,
$$

then $N_{\theta}(E, A, \Lambda)=N_{\theta}(E, A, \Lambda, \mathscr{M})$.

Proof. If $\lim _{v \rightarrow \infty} \inf _{i}\left(M_{i}\left(v / \rho^{(i)}\right) / v / \rho^{(i)}\right)>0$ for some $\rho^{(i)}>$ 0 , then there exists a number $\gamma>0$ such that

$$
M_{i}\left(\frac{v}{\rho^{(i)}}\right) \geq \gamma\left(\frac{v}{\rho^{(i)}}\right) \quad \forall v>0 \text { and some } \rho^{(i)}>0 .
$$

Let $x \in N_{\theta}(E, A, \Lambda, \mathscr{M})$ and $x \stackrel{\Lambda}{\rightarrow} s$, where $s=\left(s_{1}, s_{2}, \ldots\right) \in E$, $e_{i} \in \mathbb{C}$. Then clearly

$$
\begin{gathered}
\frac{1}{h_{r} \sum_{i \in I_{r}}}\left[M_{i}\left(\frac{\left\|A_{i}\left(\Lambda_{i}(x)-s_{i} e_{i}\right)\right\|}{\rho^{(i)}}\right)\right] \\
\geq \frac{1}{h_{r}} \sum_{i \in I_{r}} u_{i}\left[\gamma\left(\frac{\left\|A_{i}\left(\Lambda_{i}(x)-s_{i} e_{i}\right)\right\|}{\rho^{(i)}}\right)\right] \\
=\gamma \frac{1}{h_{r}} \sum_{i \in I_{r}}\left(\frac{\left\|A_{i}\left(\Lambda_{i}(x)-s_{i} e_{i}\right)\right\|}{\rho^{(i)}}\right) .
\end{gathered}
$$

Hence $x \in N_{\theta}(E, A, \Lambda)$. This completes the proof.

\section{Relation between the Spaces $\left|\sigma_{1}(E, A, \Lambda)\right|$ and $N_{\theta}(E, A, \Lambda)$}

In this section of the paper we study relation between the spaces $\left|\sigma_{1}(E, A, \Lambda)\right|$ and $N_{\theta}(E, A, \Lambda)$.

Lemma 6. $\left|\sigma_{1}(E, A, \Lambda)\right|^{0} \subset N_{\theta}(E, A, \Lambda)$ if and only if $\lim \inf _{r} q_{r}>1$. 
Proof. First suppose that $\liminf _{r} q_{r}>1$. Then there exist $\delta>$ 0 such that $1+\delta \leq q_{r}$ for all $r \geq 1$. Let $x \in\left|\sigma_{1}(E, A, \Lambda)\right|^{0}$. Then

$$
\begin{aligned}
\frac{1}{h_{r}} \sum_{i \in I_{r}}\left\|A_{i}\left(\Lambda_{i}(x)\right)\right\|= & \frac{1}{h_{r}} \sum_{i=1}^{k_{r}}\left\|A_{i}\left(\Lambda_{i}(x)\right)\right\| \\
& -\frac{1}{h_{r}} \sum_{i=1}^{k_{r-1}}\left\|A_{i}\left(\Lambda_{i}(x)\right)\right\| \\
= & \frac{k_{r}}{h_{r}}\left(\frac{1}{k_{r}} \sum_{i=1}^{k_{r}}\left\|A_{i}\left(\Lambda_{i}(x)\right)\right\|\right) \\
& -\frac{k_{r-1}}{h_{r}}\left(\frac{1}{k_{r-1}} \sum_{i=1}^{k_{r-1}}\left\|A_{i}\left(\Lambda_{i}(x)\right)\right\|\right) .
\end{aligned}
$$

Now, $h_{r}=k_{r}-k_{r-1}$. So we have

$$
\frac{k_{r}}{h_{r}}=\frac{k_{r}}{k_{r}-k_{r-1}}=\frac{q_{r}}{q_{r}-1}=1+\frac{1}{q_{r}-1} \leq 1+\frac{1}{\delta}=\frac{\delta+1}{\delta} .
$$

Also

$$
\frac{k_{r-1}}{h_{r}}=\frac{k_{r-1}}{k_{r}-k_{r-1}}=\frac{1}{q_{r}-1} \leq \frac{1}{\delta} .
$$

Since $x \in\left|\sigma_{1}(E, A, \Lambda)\right|^{0}$, then

$$
\frac{1}{k_{r}} \sum_{i=1}^{k_{r}}\left\|A_{i}\left(\Lambda_{i}(x)\right)\right\| \longrightarrow 0, \quad \frac{1}{k_{r-1}} \sum_{i=1}^{k_{r-1}}\left\|A_{i}\left(\Lambda_{i}(x)\right)\right\| \longrightarrow 0,
$$

and hence

$$
\frac{1}{h_{r}} \sum_{i \in I_{r}}\left\|A_{i}\left(\Lambda_{i}(x)\right)\right\| \longrightarrow 0
$$

that is, $x \in N_{\theta}^{0}(E, A, \Lambda)$. By linearity, it follows that $\left|\sigma_{1}(E, A, \Lambda)\right|^{0} \subset N_{\theta}(E, A, \Lambda)$.

Next, suppose that $\lim _{\inf _{r}} q_{r}=1$. Since $\theta$ is lacunary we can select a subsequence $k_{r_{j}}$ of $\theta$ such that

$$
\frac{k_{r_{j}}}{k_{r_{j}-1}}<1+\frac{1}{j}, \quad \frac{k_{r_{j}-1}}{k_{r_{j-1}}}>j,
$$

where $r_{j} \geq r_{j-1}+2$. Define $x=\left(x_{i}\right)$ by

$$
\Lambda_{i}(x)= \begin{cases}e_{i}, & \text { if } i \in I_{r_{j}}, \text { for some } j=1,2, \ldots, \\ \theta, & \text { otherwise, }\end{cases}
$$

where $\left\|e_{i}\right\|=1$ and let $A=I$, and then for any $L=$ $\left(L_{1}, L_{2}, \ldots\right) \in E, e_{i} \in \mathbb{C}$,

$$
\begin{aligned}
\frac{1}{h_{r_{j}}} \sum_{i \in I_{r}}\left(\frac{\left\|A_{i}\left(\Lambda_{i}(x)-L\right)\right\|}{\rho}\right) & =\frac{\left\|e_{i}-L_{i} e_{i}\right\|}{\rho} \\
& =\frac{\left\|1-L_{i}\right\|}{\rho} \text { for } j=1,2, \ldots,
\end{aligned}
$$

$$
\frac{1}{h_{r}} \sum_{i \in I_{r}}\left(\frac{\left\|A_{i}\left(\Lambda_{i}(x)\right)\right\|}{\rho}\right)=\frac{\left\|e_{i}\right\|}{\rho}=\frac{1}{\rho} .
$$

So, $x \notin N_{\theta}(E, A, \Lambda)$. But $x$ is strongly Cesàro-summable, since if $t$ is sufficiently large integer we can find the unique $j$ for which $k_{r_{j}-1}<t \leq k_{r_{j+1}-1}$ and hence

$$
\begin{aligned}
\frac{1}{t} \sum_{i=1}^{t}\left(\left\|A_{i}\left(\Lambda_{i}(x)\right)\right\|\right) & <\frac{1}{k_{r_{j}-1}} \sum_{i=1}^{t} 1 \\
& \leq \frac{1}{k_{r_{j}-1}} k_{r_{j}} \leq \frac{k_{r_{j}-1}+h_{r_{j}}}{k_{r_{j}-1}} \\
& <\frac{1}{j}+\frac{1}{j}=\frac{2}{j}, \quad \text { as } t \longrightarrow \infty,
\end{aligned}
$$

and it follows that also $j \rightarrow \infty$. Hence $x \in\left|\sigma_{1}(E, A, \Lambda)\right|^{0}$.

Lemma 7. $N_{\theta}(E, A, \Lambda) \subset\left|\sigma_{1}(E, A, \Lambda)\right|$ if and only if $\lim \sup _{r} q_{r}<\infty$.

Proof. First suppose that if $\lim _{\sup _{r}} q_{r}<\infty$, there exists $M>$ 0 such that $q_{r}<M$ for all $r \geq 1$. Let $x \in N_{\theta}(E, A, \Lambda)$ and $\epsilon>0$. Then

$$
\lim _{r \rightarrow \infty} \frac{1}{h_{r}} \sum_{i \in I_{r}}\left(\frac{\left\|A_{i}\left(\Lambda_{i}(x)\right)\right\|}{\rho}\right)=0 \quad \text { for some } \rho>0 .
$$

Then we can find $R>0$ and $K>0$ such that

$$
\sup _{j \geq R} \frac{1}{h_{j}} \sum_{I_{j}}\left(\frac{\left\|A_{i}\left(\Lambda_{i}(x)\right)\right\|}{\rho}\right)<\epsilon,
$$

$$
\frac{1}{h_{j}} \sum_{I_{j}}\left(\frac{\left\|A_{i}\left(\Lambda_{i}(x)\right)\right\|}{\rho}\right)<K \quad \forall i=1,2, \ldots
$$

Then if $t$ is any integer with

$$
k_{r-1} \leq t \leq k_{r}, \quad \text { where } r>R,
$$

then

$$
\begin{gathered}
\frac{1}{t} \sum_{j=1}^{t}\left(\frac{\left\|A_{i}\left(\Lambda_{i}(x)\right)\right\|}{\rho}\right) \\
\leq \frac{1}{k_{r-1}} \sum_{i=1}^{k_{r}}\left(\frac{\left\|A_{i}\left(\Lambda_{i}(x)\right)\right\|}{\rho}\right) \\
=\frac{1}{k_{r-1}}\left(\sum_{I_{1}}\left(\frac{\left\|A_{i}\left(\Lambda_{i}(x)\right)\right\|}{\rho}\right)\right. \\
+\sum_{I_{2}}\left(\frac{\left\|A_{i}\left(\Lambda_{i}(x)\right)\right\|}{\rho}\right) \\
\left.+\cdots+\sum_{I_{r}}\left(\frac{\left\|A_{i}\left(\Lambda_{i}(x)\right)\right\|}{\rho}\right)\right)
\end{gathered}
$$




$$
\begin{aligned}
& =\frac{k_{1}}{k_{r-1}} \frac{1}{h_{1}} \sum_{I_{1}}\left(\frac{\left\|A_{i}\left(\Lambda_{i}(x)\right)\right\|}{\rho}\right) \\
& +\frac{k_{2}-k_{1}}{k_{r-1}} \frac{1}{h_{2}} \sum_{I_{2}}\left(\frac{\left\|A_{i}\left(\Lambda_{i}(x)\right)\right\|}{\rho}\right) \\
& +\cdots+\frac{k_{R}-k_{R-1}}{k_{r-1}} \frac{1}{h_{R}} \sum_{I_{R}}\left(\frac{\left\|A_{i}\left(\Lambda_{i}(x)\right)\right\|}{\rho}\right) \\
& +\frac{k_{R+1}-k_{R}}{k_{r-1}} \frac{1}{h_{R+1}} \sum_{I_{R+1}}\left(\frac{\left\|A_{i}\left(\Lambda_{i}(x)\right)\right\|}{\rho}\right) \\
& +\cdots+\frac{k_{r}-k_{r-1}}{k_{r-1}} \frac{1}{h_{r}} \sum_{I_{r}}\left(\frac{\left\|A_{i}\left(\Lambda_{i}(x)\right)\right\|}{\rho}\right) \\
& \leq \frac{k_{R}}{k_{r-1}} \sup _{i \geq 1} \frac{1}{h_{i}} \sum_{I_{i}}\left(\frac{\left\|A_{i}\left(\Lambda_{i}(x)\right)\right\|}{\rho}\right) \\
& +\frac{k_{r}-k_{R}}{k_{r-1}} \frac{1}{h_{r}} \sum_{I_{r}}\left(\frac{\left\|A_{i}\left(\Lambda_{i}(x)\right)\right\|}{\rho}\right) \\
& <K \frac{k_{R}}{k_{r-1}}+\epsilon\left(q_{r}-\frac{k_{R}}{k_{r-1}}\right) \\
& <K \frac{k_{R}}{k_{r-1}}+\epsilon q_{r} \\
& <K \frac{k_{R}}{k_{r-1}}+\epsilon M
\end{aligned}
$$

Since $k_{r-1} \rightarrow \infty$ as $r \rightarrow \infty$, it follows that

$$
\frac{1}{t} \sum_{j=1}^{t}\left(\frac{\left\|A_{i}\left(\Lambda_{i}(x)\right)\right\|}{\rho}\right) \longrightarrow 0
$$

and hence $x \in\left|\sigma_{1}(E, A, \Lambda)\right|$.

Next, suppose that $\lim \sup _{r} q_{r}=\infty$. We construct a sequence in $N_{\theta}(E, A, \Lambda)$ that is not Cesaro $\Lambda$-summable. By the idea of Freedman et al. [12] we can construct a subsequence $k_{r_{j}}$ of the lacunary sequence $\theta=\left(k_{r}\right)$ such that $q_{r_{j}}>j$, and then define a bounded difference sequence $x$ by

$$
\Lambda_{i}(x)= \begin{cases}e_{i}, & \text { if } k_{r_{j}-1}<i<2 k_{r_{j}-1} \\ \theta, & \text { otherwise }\end{cases}
$$

where $\left\|e_{i}\right\|=1$. Let $A=I$ and $\rho=1$. Then,

$$
\frac{1}{h_{r_{j}}} \sum_{I_{r_{j}}}\left(\left\|A_{i}\left(\Lambda_{i}(x)\right)\right\|\right)=\frac{2 k_{r_{j}-1}-k_{r_{j}-1}}{k_{r_{j}}-k_{r_{j}-1}}=\frac{k_{r_{j}-1}}{k_{r_{j}}-k_{r_{j}-1}}<\frac{1}{j-1}
$$

and if $r \neq r_{j}$,

$$
\frac{1}{h_{r_{j}}} \sum_{I_{r_{j}}}\left(\left\|A_{i}\left(\Lambda_{i}(x)\right)\right\|\right)=0
$$

Thus $x \in N_{\theta}(E, A, \Lambda)$. For the above sequence and for $i=$ $1,2, \ldots, k_{r_{j}}$

$$
\begin{aligned}
\frac{1}{k_{r_{j}}} \sum_{i}\left(\left\|A_{i}\left(\Lambda_{i}(x)-e_{i}\right)\right\|\right) & >\frac{1}{k_{r_{j}}}\left(2 k_{r_{j}-1}-k_{r_{j}-1}\right) \\
& =1-\frac{2}{q_{r_{j}}}>1-\frac{2}{j},
\end{aligned}
$$

this converges to 1 , but for $i=1,2, \ldots, 2 k_{r_{j}-1}$,

$$
\frac{2}{k_{r_{j-1}}} \sum_{i}\left(\left\|A_{i}\left(\Lambda_{i}(x)\right)\right\|\right) \geq \frac{k_{r_{j}-1}}{2 k_{r_{j}-1}}=\frac{1}{2} .
$$

It proves that $x \notin\left|\sigma_{1}(E, A, \Lambda)\right|$, since any sequence in $\left|\sigma_{1}(E, A, \Lambda)\right|$ consisting of $\theta$ 's and $e_{i}$ 's has a limit only 0 or 1.

Theorem 8. Let $\theta$ be a lacunary sequence. Then $\left|\sigma_{1}(E, A, \Lambda)\right|=$ $N_{\theta}(E, A, \Lambda)$ if and only if $1 \leq \liminf _{r} q_{r} \leq \lim \sup _{r} q_{r}<\infty$.

Proof. The proof of the theorem follows from Lemmas 6 and 7.

\section{Statistical Convergence}

The notion of statistical convergence was introduced by Fast [16] and Schoenberg [17] independently. Over the years and under different names, statistical convergence has been discussed in the theory of Fourier analysis, ergodic theory, and number theory. Later on, it was further investigated from the sequence space point of view and linked with summability theory by Fridy [18], Connor [19], Šalát [20], Mursaleen and Edely [21], Isk [22], Mohiuddine and Alghamdi [23], Hazarika et al. [24], Kolk [25], Maddox [26], Alotaibi and Mursaleen [27], Mohiuddine et al. [28], Mohiuddine and Aiyub [29], and many others. In recent years, generalizations of statistical convergence have appeared in the study of strong integral summability and the structure of ideals of bounded continuous functions on locally compact spaces. Statistical convergence and its generalizations are also connected with subsets of the Stone-Čech compactification of natural numbers. Moreover, statistical convergence is closely related to the concept of convergence in probability. The notion depends on the density of subsets of the set $\mathbb{N}$ of natural numbers.

A subset $E$ of $\mathbb{N}$ is said to have the natural density $\delta(E)$ if the following limit exists:

$$
\delta(E)=\lim _{n \rightarrow \infty} \frac{1}{n} \sum_{k=1}^{n} \chi_{E}(k)
$$

where $\chi_{E}$ is the characteristic function of $E$. It is clear that any finite subset of $\mathbb{N}$ has zero natural density and $\delta\left(E^{c}\right)=$ $1-\delta(E)$.

A sequence $x=\left(x_{k}\right)$ is said to be statistically convergent to the number $L$ if for every $\epsilon>0$

$$
\lim _{n \rightarrow \infty} \frac{1}{n}\left|\left\{k \leq n:\left|x_{k}-L\right| \geq \epsilon\right\}\right|=0
$$


Bilgin [14] also introduced the concept of statistical convergence in $N_{0}(A, F)$ and proved some inclusion relation.

Let $\theta$ be a lacunary sequence and let $A=\left(a_{i k}\right)$ be an infinite matrix of complex numbers. Then a sequence $x \in N_{\theta}(E, A, \Lambda, \mathscr{M})$ is said to be $\Lambda$-lacunary $A$-statistically convergent to a number $s=\left(s_{1}, s_{2}, \ldots\right) \in E, e_{i} \in \mathbb{C}$ if for any $\epsilon>0$,

$$
\lim _{r \rightarrow \infty} \frac{1}{h_{r}}\left|\Lambda_{k} A_{0}(\epsilon)\right|=0
$$

where

$$
\Lambda A_{0}(\epsilon)=\left\{i \in I_{r}: M_{i}\left(\frac{\left\|A_{i}\left(\Lambda_{k}(x)-s_{i} e_{i}\right)\right\|}{\rho^{(i)}}\right) \geq \epsilon\right\} .
$$

We denote it as $x \stackrel{\Lambda \text {-stat }}{\longrightarrow} s$. The vertical bar denotes the cardinality of the set. The set of all $\Lambda$-lacunary $A$-statistical convergent sequences is denoted by $S_{\theta}(E, A, \Lambda, \mathscr{M})$.

In this section we study some relation between the spaces $S_{\theta}(E, A, \Lambda, \mathscr{M})$ and $N_{\theta}(E, A, \Lambda, \mathscr{M})$.

Theorem 9. Let $\mathscr{M}=\left(M_{i}\right)$ be a Musielak-Orlicz function and let $\left(M_{i}\right)$ be pointwise convergent. Then $N_{\theta}(E, A, \Lambda, M) \subset$ $S_{\theta}(E, A, \Lambda, \mathscr{M})$ if and only if $\lim _{i} M_{i}\left(v / \rho^{(i)}\right)>0$ for some $v>0, \rho^{(i)}>0$.

Proof. Let $\epsilon>0$ and $x \in N_{\theta}(E, A, \Lambda, \mathscr{M})$. Let $x \stackrel{\Lambda}{\rightarrow} s$, where $s=\left(s_{1}, s_{2}, \ldots\right) \in E, e_{i} \in \mathbb{C}$. Since $\lim _{i} M_{i}(v / \rho)>0$, there exists a number $c>0$ such that

$$
M_{i}\left(\frac{v}{\rho}\right) \geq c \quad \text { for } v>\epsilon .
$$

Let

$$
I_{r}^{1}=\left\{i \in I_{r}:\left[M_{i}\left(\frac{\left\|A_{i}\left(\Lambda_{i}(x)-s_{i} e_{i}\right)\right\|}{\rho^{(i)}}\right)\right] \geq \epsilon\right\} .
$$

Then

$$
\begin{aligned}
\frac{1}{h_{r}} \sum_{i \in I_{r}} & {\left[M_{i}\left(\frac{\left\|A_{i}\left(\Lambda_{i}(x)-s_{i} e_{i}\right)\right\|}{\rho^{(i)}}\right)\right] } \\
& \geq \frac{1}{h_{r}} \sum_{i \in I_{r}^{1}}\left[M_{i}\left(\frac{\left\|A_{i}\left(\Lambda_{i}(x)-s_{i} e_{i}\right)\right\|}{\rho^{(i)}}\right)\right] \\
& \geq c \frac{1}{h_{r}}\left|\Lambda_{k} A_{0}(\epsilon)\right| .
\end{aligned}
$$

Hence it follows that $x \in S_{\theta}(E, A, \Lambda, \mathscr{M})$.

Conversely, let us assume that the condition does not hold good. Then there is a number $v>0$ such that $\lim _{i} M_{i}(v / \rho)=0$ for some $\rho>0$. Now, we select a lacunary sequence $\theta=\left(k_{r}\right)$ such that $M_{i}(v / \rho)<2^{-r}$ for any $i>k_{r}$.

Let $A=I$, and define the sequence $x$ by putting

$$
\Lambda_{i}(x)= \begin{cases}v, & \text { if } k_{r-1}<i \leq \frac{k_{r}+k_{r-1}}{2}, \\ \theta, & \text { if } \frac{k_{r}+k_{r-1}}{2}<i \leq k_{r} .\end{cases}
$$

Therefore,

$$
\begin{aligned}
\frac{1}{h_{r}} \sum_{i \in I_{r}} M_{i}\left(\frac{\left\|\Lambda_{i}(x)\right\|}{\rho^{(i)}}\right) \\
\quad=\frac{1}{h_{r}} \sum_{k_{r-1}<i \leq\left(k_{r}+k_{r-1}\right) / 2} M_{i}\left(\frac{v}{\rho^{(i)}}\right) \\
<\frac{1}{h_{r}} \frac{1}{2^{r-1}}\left[\frac{k_{r}+k_{r-1}}{2}-k_{r-1}\right] \\
=\frac{1}{2^{r}} \longrightarrow 0 \text { as } r \longrightarrow \infty .
\end{aligned}
$$

Thus, we have $x \in N_{\theta}^{0}(E, A, \Lambda, \mathscr{M})$. But

$$
\begin{aligned}
& \lim _{r \rightarrow \infty} \frac{1}{h_{r}}\left|\left\{i \in I_{r}: M_{i}\left(\frac{\left\|\Lambda_{i}(x)\right\|}{\rho^{(i)}}\right) \geq \epsilon\right\}\right| \\
& =\lim _{r \rightarrow \infty} \frac{1}{h_{r}}\left|\left\{i \epsilon\left(k_{r-1}, \frac{k_{r}+k_{r-1}}{2}\right): M_{i}\left(\frac{v}{\rho^{(i)}}\right) \geq \epsilon\right\}\right| \\
& =\lim _{r \rightarrow \infty} \frac{1}{h_{r}} \frac{k_{r}-k_{r-1}}{2}=\frac{1}{2} .
\end{aligned}
$$

So $x \notin S_{\theta}(E, A, \Lambda, \mathscr{M})$.

Theorem 10. Let $\mathscr{M}=\left(M_{i}\right)$ be a Musielak-Orlicz function. Then $S_{\theta}(E, A, \Lambda, \mathscr{M}) \subset N_{\theta}(E, A, \Lambda, \mathscr{M})$ if and only if $\sup _{v} \sup _{i} M_{i}(v / \rho)<\infty$.

Proof. Let $x \in S_{\theta}(E, A, \Lambda, \mathscr{M})$ and $x \stackrel{\Lambda \text {-stat }}{\longrightarrow} s$. Suppose $h(v)=$ $\sup _{i} M_{i}(v / \rho)$ and $h=\sup _{v} h(v)$. Let

$$
I_{r}^{2}=\left\{i \in I_{r}: M_{i}\left(\frac{\left\|A_{i}\left(\Lambda_{i}(x)-s_{i} e_{i}\right)\right\|}{\rho^{(i)}}\right)<\epsilon\right\} .
$$

Now, $M_{i}(v) \leq h$ for all $i, v>0$. So

$$
\begin{aligned}
\frac{1}{h_{r}} \sum_{i \in I_{r}} & {\left[M_{i}\left(\frac{\left\|A_{i}\left(\Lambda_{i}(x)-s_{i} e_{i}\right)\right\|}{\rho^{(i)}}\right)\right] } \\
= & \frac{1}{h_{r}} \sum_{i \in I_{r}^{1}}\left[M_{i}\left(\frac{\left\|A_{i}\left(\Lambda_{i}(x)-s_{i} e_{i}\right)\right\|}{\rho^{(i)}}\right)\right] \\
& +\frac{1}{h_{r_{i}}} \sum_{i \in I_{r}^{2}}\left[M_{i}\left(\frac{\left\|A_{i}\left(\Lambda_{i}(x)-s_{i} e_{i}\right)\right\|}{\rho^{(i)}}\right)\right] \\
\leq & h \frac{1}{h_{r}}\left|\Lambda_{k} A_{0}(\epsilon)\right|+h(\epsilon) .
\end{aligned}
$$

Hence, as $\epsilon \rightarrow 0$, it follows that $x \in N_{\theta}(E, A, \Lambda, \mathscr{M})$.

Conversely, suppose that

$$
\sup _{v} \sup _{i} M_{i}\left(\frac{v}{\rho}\right)=\infty .
$$

Then we have

$$
0<v_{1}<v_{2}<\cdots<v_{r-1}<v_{r}<\cdots,
$$


so that $M_{k_{r}}\left(v_{r} / \rho\right) \geq h_{r}$ for $r \geq 1$. Let $A=I$. We set a sequence $x=\left(x_{i}\right)$ by

$$
\Lambda_{i}(x)= \begin{cases}v_{r}, & \text { if } i=k_{r} \text { for some } r=1,2, \ldots, \\ \theta, & \text { otherwise. }\end{cases}
$$

Then

$$
\lim _{r \rightarrow \infty} \frac{1}{h_{r}}\left|\left\{i \in I_{r}:\left[M_{i}\left(\frac{\left\|\Lambda_{i}(x)\right\|}{\rho^{(i)}}\right)\right] \geq \epsilon\right\}\right|=\lim _{r \rightarrow \infty} \frac{1}{h_{r}}=0 .
$$

Hence $x \stackrel{\Lambda \text {-stat }}{\longrightarrow} 0$ and hence $x \in S_{\theta}(E, A, \Lambda, \mathscr{M})$.

But

$$
\begin{aligned}
& \lim _{r \rightarrow \infty} \frac{1}{h_{r}} \sum_{i \in I_{r}}\left[M_{i}\left(\frac{\left\|A_{i}\left(\Lambda_{i}(x)-s_{i} e_{i}\right)\right\|}{\rho^{(i)}}\right)\right] \\
& =\lim _{r \rightarrow \infty} \frac{1}{h_{r}}\left[M_{k_{r}}\left(\frac{\left\|v_{r}-s_{i} e_{i}\right\|}{\rho^{(i)}}\right)\right] \\
& \geq \lim _{r \rightarrow \infty} \frac{1}{h_{r}} h_{r}=1 .
\end{aligned}
$$

So, $x \notin N_{\theta}(E, A, \Lambda, \mathscr{M})$.

\section{Conflict of Interests}

The authors declare that there is no conflict of interests regarding the publication of this paper.

\section{References}

[1] J. Lindenstrauss and L. Tzafriri, "On Orlicz sequence spaces," Israel Journal of Mathematics, vol. 10, pp. 379-390, 1971.

[2] L. Maligranda, Orlicz Spaces and Interpolation, Seminars in Mathematics 5, Polish Academy of Science, 1989.

[3] J. Musielak, Orlicz Spaces and Modular Spaces, vol. 1034 of Lecture Notes in Mathematics, Springer, Berlin, Germany, 1983.

[4] A. Wilansky, Summability through Functional Analysis, vol. 85, North-Holland, Amsterdam, Netherlands, 1984.

[5] M. Mursaleen and A. K. Noman, "On some new sequence spaces of non absolute type related to the spaces $l_{p}$ and $l_{\infty}$ II," Mathematical Communications, vol. 16, pp. 383-398, 2011.

[6] S. D. Parashar and B. Choudhary, "Sequence spaces defined by Orlicz functions," Indian Journal of Pure and Applied Mathematics, vol. 25, no. 4, pp. 419-428, 1994.

[7] K. Raj and S. K. Sharma, "Some sequence spaces in 2-normed spaces defined by Musielak-Orlicz function," Acta Universitatis Sapientiae: Mathematica, vol. 3, no. 1, pp. 97-109, 2011.

[8] K. Raj and S. K. Sharma, "Some generalized difference double sequence spaces defined by a sequence of Orlicz-functions," Cubo, vol. 14, no. 3, pp. 167-189, 2012.

[9] K. Raj and S. K. Sharma, "Some multiplier sequence spaces defined by a Musielak-Orlicz function in $n$-normed spaces," New Zealand Journal of Mathematics, vol. 42, pp. 45-56, 2012.

[10] A. Gökhan, M. Et, and M. Mursaleen, "Almost lacunary statistical and strongly almost lacunary convergence of sequences of fuzzy numbers," Mathematical and Computer Modelling, vol. 49, no. 3-4, pp. 548-555, 2009.
[11] M. Gungor and M. Et, " $\Delta^{T}$-strongly almost summable sequences defined by Orlicz functions," Indian Journal of Pure and Applied Mathematics, vol. 34, no. 8, pp. 1141-1151, 2003.

[12] A. R. Freedman, J. J. Sember, and M. Raphael, "Some Cesàrotype summability spaces," Proceedings of the London Mathematical Society, vol. 37, no. 3, pp. 508-520, 1978.

[13] T. Bilgin, "Lacunary strong $A$-convergence with respect to a modulus," Studia Universitatis Babeş-Bolyai, vol. 46, no. 4, pp. 39-46, 2001.

[14] T. Bilgin, "Lacunary strong A-convergence with respect to a sequence of modulus functions," Applied Mathematics and Computation, vol. 151, no. 3, pp. 595-600, 2004.

[15] M. Mursaleen and A. K. Noman, "On some new sequence spaces of non-absolute type related to the spaces $l_{p}$ and $l_{\infty} \mathrm{I}$," Filomat, vol. 25, no. 2, pp. 33-51, 2011.

[16] H. Fast, "Sur la convergence statistique," Colloquium Mathematicae, vol. 2, pp. 241-244, 1951.

[17] I. J. Schoenberg, "The integrability of certain functions and related summability methods," The American Mathematical Monthly, vol. 66, pp. 361-375, 1959.

[18] J. A. Fridy, “On statistical convergence," Analysis, vol. 5, no. 4, pp. 301-313, 1985.

[19] J. S. Connor, "A topological and functional analytic approach to statistical convergence," in Applied and Numerical Harmonic Analysis, vol. 8 of Analysis of Divergence, pp. 403-413, 1999.

[20] T. Šalát, "On statistically convergent sequences of real numbers," Mathematica Slovaca, vol. 30, no. 2, pp. 139-150, 1980.

[21] M. Mursaleen and O. H. H. Edely, "Generalized statistical convergence," Information Sciences, vol. 162, no. 3-4, pp. 287294, 2004.

[22] M. Isk, "On statistical convergence of generalized difference sequences," Soochow Journal of Mathematics, vol. 30, no. 2, pp. 197-205, 2004.

[23] S. A. Mohiuddine and M. A. Alghamdi, "Statistical summability through a lacunary sequence in locally solid Riesz spaces," Journal of Inequalities and Applications, vol. 2012, article 225, 2012.

[24] B. Hazarika, S. A. Mohiuddine, and M. Mursaleen, "Some inclusion results for lacunary statistical convergence in locally solid Riesz spaces," Iranian Journal of Science Technology, vol. 38, no. A1, pp. 61-68, 2014.

[25] E. Kolk, “The statistical convergence in Banach spaces," Acta et Commentationes Universitatis Tartuensis, vol. 928, pp. 41-52, 1991.

[26] I. J. Maddox, "Statistical convergence in a locally convex space," Mathematical Proceedings of the Cambridge Philosophical Society, vol. 104, no. 1, pp. 141-145, 1988.

[27] A. Alotaibi and M. Mursaleen, "Statistical convergence in random paranormed space," Journal of Computational Analysis and Applications, vol. 17, no. 2, pp. 297-304, 2014.

[28] S. A. Mohiuddine, K. Raj, and A. Alotaibi, "Some paranormed double difference sequence spaces for Orlicz functions and bounded-regular matrices," Abstract and Applied Analysis, vol. 2014, Article ID 419064, 10 pages, 2014.

[29] S. A. Mohiuddine and M. Aiyub, "Lacunary statistical convergence in random 2-normed spaces," Applied Mathematics \& Information Sciences, vol. 6, no. 3, pp. 581-585, 2012. 


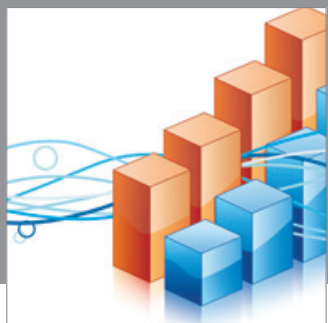

Advances in

Operations Research

mansans

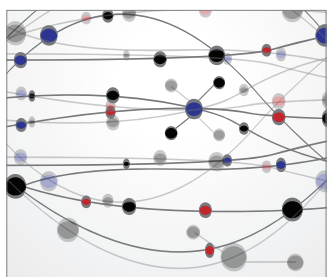

The Scientific World Journal
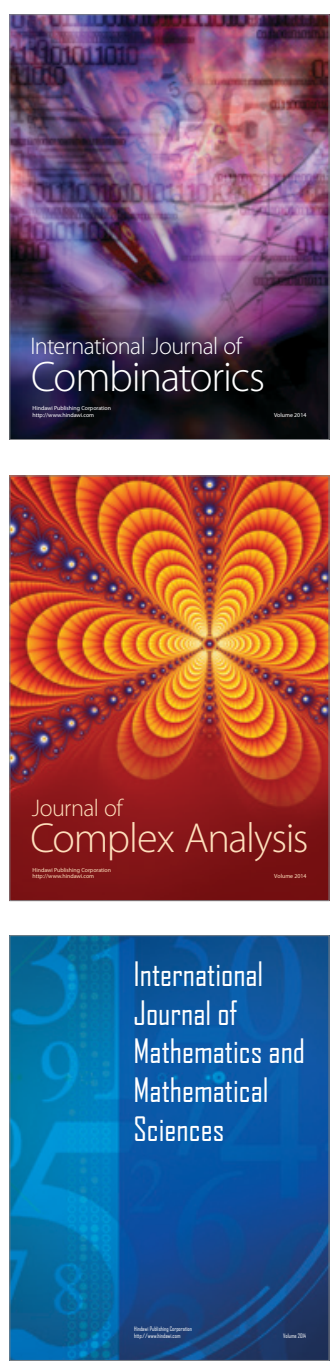
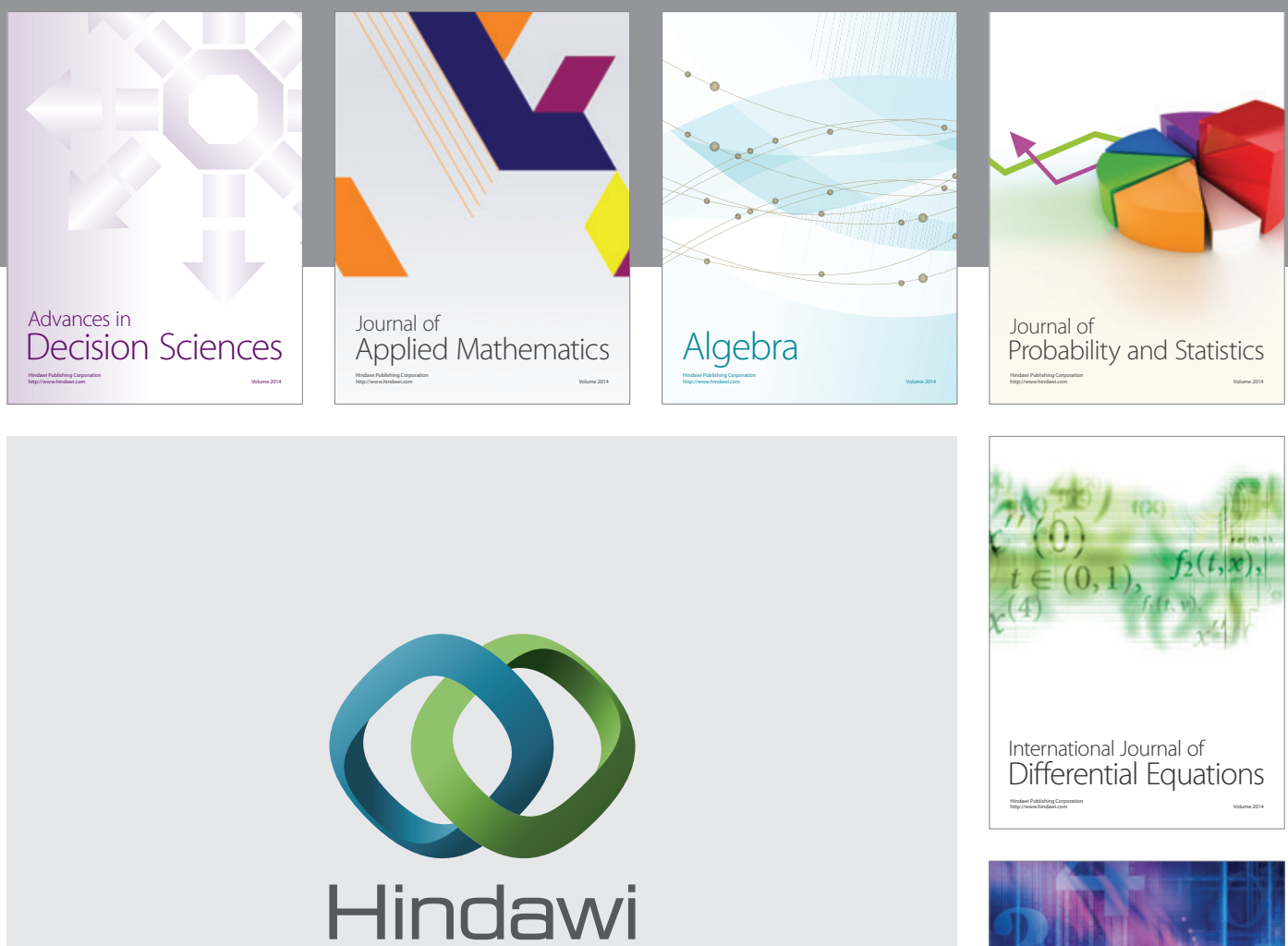

Submit your manuscripts at http://www.hindawi.com
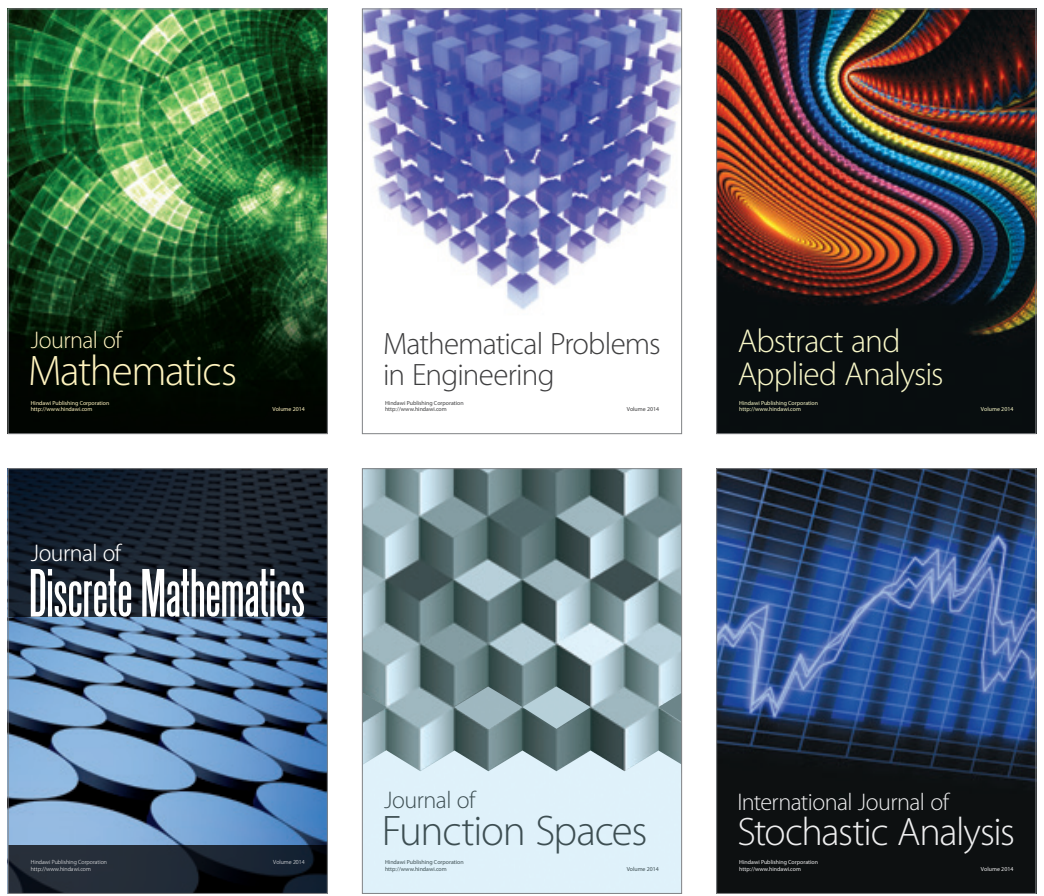

Journal of

Function Spaces

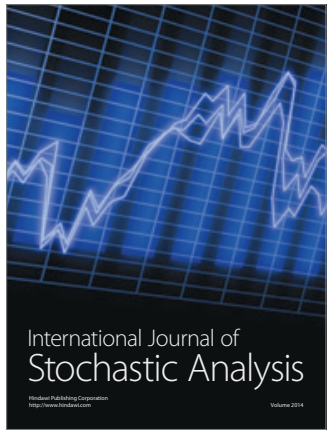

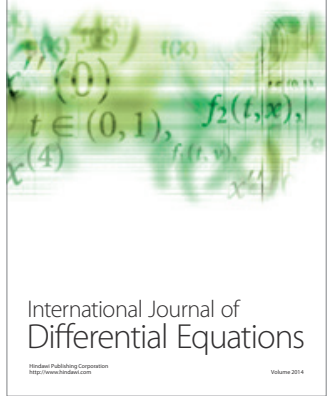
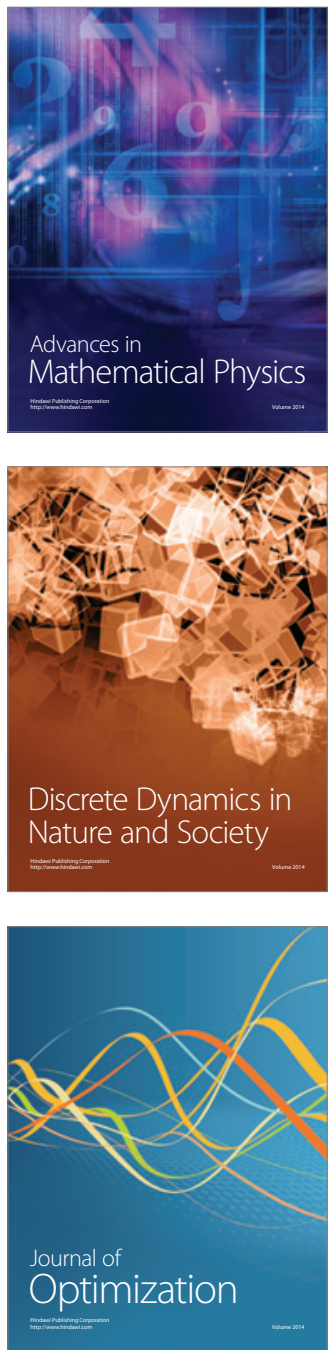